Check for updates

Cite this: Nanoscale Adv., 2019, 1, 1489

\section{Sulfur-doped graphene/transition metal dichalcogenide heterostructured hybrids with electrocatalytic activity toward the hydrogen evolution reaction $\uparrow$}

\author{
Antonia Kagkoura, ${ }^{a}$ Mario Pelaez-Fernandez, ${ }^{\text {b }}$ Raul Arenal ${ }^{\text {bcd }}$ \\ and Nikos Tagmatarchis (iD *a
}

\begin{abstract}
A facile route for the preparation of molybdenum disulfide $\left(\mathrm{MoS}_{2}\right)$ and tungsten disulfide $\left(\mathrm{WS}_{2}\right)$, uniformly deposited onto sulfur-doped graphene (SG), is reported. The realization of the SG/MoS 2 and SG/WS 2 heterostructured hybrids was accomplished by employing microwave irradiation for the thermal decomposition of ammonium tetrathiomolybdate and tetrathiotungstate, respectively, in the presence of SG. Two different weight ratios between SG and the inorganic species were used, namely $3: 1$ and $1: 1$, yielding SG/MoS $2(3: 1), S G / M o S_{2}(1: 1), S G / W S_{2}(3: 1)$ and SG/WS $2(1: 1)$. SG and all newly developed hybrid materials were characterized by ATR-IR and Raman spectroscopy, TGA, HR-TEM and EELS. The electrocatalytic activity of the $\mathrm{SG} / \mathrm{MoS}_{2}$ and $\mathrm{SG} / \mathrm{WS}_{2}$ heterostructured hybrids was examined against the hydrogen evolution reaction (HER) and it was found that the presence of SG not only significantly improved the catalytic activity of $\mathrm{MOS}_{2}$ and $\mathrm{WS}_{2}$ but also made it comparable to that of commercial $\mathrm{Pt} / \mathrm{C}$. Specifically, hybrids containing higher amounts of SG, namely SG/MoS $(3: 1)$ and SG/WS $2(3: 1)$, exhibited extremely low onset overpotentials of 26 and $140 \mathrm{mV} v \mathrm{~s}$. RHE, respectively. The latter results highlighted the beneficial role of SG as a substrate for immobilizing $\mathrm{MoS}_{2}$ and $\mathrm{WS}_{2}$ and stressed its significance for achieving optimum electrocatalytic performance toward the HER. Finally, examination of the Tafel slopes as extracted from the electrocatalytic polarization curves, manifested the adsorption of hydrogen as the rate-limiting step for SG/MoS $(3: 1)$, while for SG/WS $(3: 1)$ the electrochemical desorption of adsorbed hydrogen atoms to generate hydrogen was revealed to be the rate-limiting step.
\end{abstract}

Received 5th August 2018

Accepted 30th January 2019

DOI: $10.1039 / \mathrm{c} 8 \mathrm{na00130h}$

rsc.li/nanoscale-advances high cost and low natural abundance prevent their large-scale use. Hence, the increase of the catalytic activity of non Ptbased, inexpensive and abundant catalysts is an extremely important, yet challenging issue. In recent years, research has focused on non-noble electrocatalysts for the Hydrogen Evolution Reaction (HER), with transition metal dichalcogenides (TMDs) rapidly evolving as promising materials with low cost and high chemical stability.

In general, TMDs are two-dimensional layered materials composed of stacks of atomic metal layers sandwiched by chalcogen layers, in which the individual chalcogen-metalchalcogen layers weakly interact each other. These materials are considered to be the inorganic analogues of graphene, as the weak van der Waals forces between the layers can be easily overcome when exfoliated from the bulk, leading to few or even monolayered sheets. Molybdenum and tungsten disulfide, $\mathrm{MoS}_{2}$ and $\mathrm{WS}_{2}$, respectively, as typical examples of TMDs, have been exfoliated from the bulk by diverse wet chemistry approaches, mainly assisted by sonication, ${ }^{1-5}$ via a top-down approach. The exfoliated TMDs exhibit dramatically different properties from the bulk material, ${ }^{6}$ and their novel physical and 
electronic properties ${ }^{7,8}$ make them suitable for energy related applications. ${ }^{9}$

Recently, TMDs have started to be used as effective materials for the HER, owing to their appealing electrocatalytic properties and based on the improvement of the energy conversion efficiency through harvesting a higher current density at a lower overpotential. ${ }^{\mathbf{1 0 - 1 8}}$ The HER activity of TMDs is directly related with exposed edges, in contrast to the catalytically inert basal planes. ${ }^{19}$ Hence, in order to improve the electrocatalytic performance of TMDs, the density of active edges should be increased. This can be achieved by edge and/or defect engineering, based on unsaturated chalcogen atoms at the edges ${ }^{15,20-22}$ and/or by promoting the electron transport efficiency between the electrode and the electrocatalyst. Furthermore, the population of defect sites can be adjusted by adopting a synthetic bottom-up approach of TMDs coupled with the presence of an additional component playing the role of the electrocatalyst substrate. ${ }^{23}$ Specifically, incorporating TMDs on supports with high surface area can provide more active edge sites for electrocatalysis, while by employing highly conductive supports, such as graphene, fast electron transport is guaranteed.

Graphene, due to its large surface area, excellent electrical conductivity and high chemical stability, has been widely employed as supporting material for electrocatalysts in general $^{24}$ and for TMDs in particular. ${ }^{18,25-32}$ In the same context, introduction of heteroatoms within the $\mathrm{sp}^{2}$ hybridized carbon network of graphene alters its electrical properties, resulting in enhanced electrocatalytic activity. The enhanced catalytic activity of graphene-doped materials is attributed to the electronegativity difference between carbon and the doping element, which polarizes the adjacent carbon atoms in the graphene lattice, hence potentially facilitating the HER. For example, this was true when $\mathrm{N}$-doped graphene/ $\mathrm{MoS}_{2}$ nanocomposites were prepared and found to exhibit high catalytic activity for the HER. ${ }^{33,34}$ However, beyond $\mathrm{N}$-doped graphene and the aforementioned results, the examination of electrocatalytic properties of other heteroatom-doped graphene materials as substrates for TMDs has yet to be realized. Particularly, focusing on sulfur-doped graphene (SG), an n-type doping effect in graphene, analogous to that observed in nitrogen-doped graphene, exists. ${ }^{35}$ However, since the difference in electronegativity between $\mathrm{S}$ and $\mathrm{C}$ as compared to that between $\mathrm{N}$ and $\mathrm{C}$ is small, an alternative mechanism for improved electrocatalytic activity is prevalent in SG. ${ }^{36}$ Briefly, incorporation of sulfur within the skeleton of graphene modifies the electronic structure of the material by inducing a non-uniform spin density distribution, which derives from the mismatch of the outermost orbitals of sulfur and carbon atoms, being responsible, along with the charge density, for the SG intrinsic electrocatalytic activity. ${ }^{37}$ Considering all the above points, the development of hybrid heterostructures, incorporating sulfur-doped graphene sheets and TMDs, as electrocatalysts is absolutely necessary and surely deserves examination.

The current study goes beyond the state of the art, by in situ growing $\mathrm{MoS}_{2}$ and $\mathrm{WS}_{2}$ onto SG, aimed at the development of efficient HER heterostructured electrocatalysts. Herein, based on the microwave-assisted thermal decomposition of the inorganic precursor species of $\mathrm{MoS}_{2}$ and $\mathrm{WS}_{2}$, namely $\left(\mathrm{NH}_{4}\right)_{2} \mathrm{MoS}_{4}$ and $\left(\mathrm{NH}_{4}\right)_{2} \mathrm{WS}_{4}$, respectively, in the presence of SG as obtained using a facile and low-cost route, the preparation of $\mathrm{SG} / \mathrm{MoS}_{2}$ and $\mathrm{SG} / \mathrm{WS}_{2}$, for two different weight ratios between the inorganic species and the SG, was accomplished. The newly developed heterostructures were characterized by Raman spectroscopy, while transmission electron microscopy (TEM) imaging allowed their morphological evaluation and electron energy loss spectroscopy (EELS) provided necessary information of the elements present in these structures. Furthermore, the SG/MoS 2 and SG/ $\mathrm{WS}_{2}$ heterostructured hybrids were tested towards the HER and it was found that the presence of SG not only significantly improved the catalytic activity of $\mathrm{MoS}_{2}$ and $\mathrm{WS}_{2}$ but also made it comparable to that of the commercial $\mathrm{Pt} / \mathrm{C}$ catalyst. The exceptional electrocatalytic functioning of $\mathrm{SG} / \mathrm{MoS}_{2}$ and $\mathrm{SG} / \mathrm{WS}_{2}$ was attributed to the following reasons: (a) the electronegativity difference between carbon and sulfur inducing n-type behaviour in S-doped graphene by polarizing the adjacent carbon atoms to sulfur in the graphene lattice and facilitating the HER, (b) the enhanced population of defect sites in TMDs, as a result of the bottom-up approach employed, (c) the intimate contact of SG with TMDs, (d) the uniform immobilization of TMDs onto the SG surface, in the absence of any organic/surfactant species, and (e) the synergetic effect between SG and TMDs.

\section{Experimental}

\section{General}

Chemicals, reagents, and solvents were purchased from SigmaAldrich and used as received. Infrared (IR) spectra were acquired on a Fourier Transform IR spectrometer (Equinox 55 from Bruker Optics) equipped with a single reflection diamond ATR accessory (DuraSamp1IR II by SensIR Technologies). Raman measurements were performed with a Renishaw confocal spectrometer at $514 \mathrm{~nm}$. The intensity ratio $I_{\mathrm{D}} / I_{\mathrm{G}}$ was obtained from the peak intensities following any baseline corrections. The data were collected and analyzed with Renishaw Wire and Origin software. Thermogravimetric analysis was performed using a TGA Q500 V20.2 Build 27 instrument (TA Instruments) in an inert nitrogen (purity > 99.999\%) atmosphere. Microwave Synthesis was undertaken in $10 \mathrm{~mL}$ vials using a CEM Discover SP Microwave System with ActiVent technology. During the synthesis, the microwave system was operated in the dynamic mode where the power was automatically adjusted to maintain the set temperature. High-resolution transmission electron microscopy (HR-TEM) and electron energy loss spectroscopy (EELS) were performed using an FEI Titan Cube TEM working at $80 \mathrm{kV}$ and equipped with a Cs image corrector and Gatan Tridiem spectrometer. The size of the analyzed area was around $200 \mathrm{~nm}$. Convergence and collection angles were 6.3 and 19.7 mrad, respectively. Typical energy resolutions (full-width at halfmaximum of the ZLP) of the measurements were better than $0.8 \mathrm{eV}$. The energy dispersion used was $0.2 \mathrm{eV}$ per channel. The EELS acquisition time was $10 \mathrm{~s}$. The TEM samples were prepared by drop-depositing samples on holey carbon thin films. Scanning electron microscopy (SEM) imaging and energy dispersive 
X-ray spectroscopy (EDS) were performed using a FE-SEM (model JSM-7610F) equipped with EDAX (X-ACT, Oxford Instruments). Electrochemical measurements were carried out at room temperature in $\mathrm{N}_{2}$-saturated $0.5 \mathrm{M} \mathrm{H}_{2} \mathrm{SO}_{4}$ in a standard threecompartment electrochemical cell by using an EG\&G Princeton Applied Research potensiostat/galvanostat (Model PARSTAT ${ }^{\circledR}$ 2273A). A platinum wire was used as the counter-electrode and as the reference electrode, a $\mathrm{Hg} / \mathrm{HgSO}_{4}\left(0.5 \mathrm{M} \mathrm{K}_{2} \mathrm{SO}_{4}\right)$ electrode was placed in a Luggin capillary. Potentials were corrected according to the relationship: $E$ vs. $\mathrm{RHE}=E$ vs. $\mathrm{Hg} / \mathrm{HgSO}_{4}+0.680$. The working electrode was a glassy carbon disk with a geometric surface area of $0.071 \mathrm{~cm}^{2}$. Linear sweep voltammetry measurements were conducted at a scan rate of $5 \mathrm{mV} \mathrm{s}^{-1}$. To prepare the catalyst ink, $4.0 \mathrm{mg}$ of the hybrid catalytic powder were dispersed in a mixture of solvents $(1 \mathrm{~mL})$ containing water, isopropanol, and $5 \%$ Nafion $(\mathrm{v} / \mathrm{v} / \mathrm{v}=4: 1: 0.02)$ and sonicated for $30 \mathrm{~min}$. The working electrode was first cleaned through polishing using 6, 3 and $1 \mathrm{~mm}$ diamond pastes, rinsed with deionized water, and sonicated in double-distilled water. Then, $3 \mu \mathrm{L}$ aliquots of the catalyst ink were cast on the electrode surface and dried at room temperature.

\section{Preparation of SG}

Initially, graphene oxide was prepared from graphite powder by following a modified Hummers' method and then SG was produced by employing a solvothermal method. In detail, $40 \mathrm{mg}$ of graphene oxide and $800 \mathrm{mg}$ of Lawesson's reagent were dispersed in diethyleneglycol methyl ether and sonicated for 30 minutes. Then the solution was refluxed at $90{ }^{\circ} \mathrm{C}$ under a $\mathrm{N}_{2}$ atmosphere for 48 hours. After that period, methanol was added and the reaction mixture was centrifuged 5 times with methanol ( 5 minutes at $4000 \mathrm{rpm}$ ). The SG was precipitated, dried at $60{ }^{\circ} \mathrm{C}$ overnight and stored in the dark.

\section{Preparation of SG/MoS ${ }_{2}$ and SG/WS}

All SG/MoS 2 and SG/WS 2 hybrids were synthesized by employing microwave irradiation conditions. To get $\mathrm{MoS}_{2}$ or $\mathrm{WS}_{2}$ the reaction mixture was prepared by adding $\left(\mathrm{NH}_{4}\right)_{2} \mathrm{MoS}_{4}$ or $\left(\mathrm{NH}_{4}\right)_{2} \mathrm{WS}_{4}(12 \mathrm{mg})$ to DMF $(3 \mathrm{~mL})$. For the preparation of the $\mathrm{SG} / \mathrm{MoS}_{2}$ or $\mathrm{SG} / \mathrm{WS}_{2}$ hybrids, $\mathrm{SG}$ was added to the reaction mixture. In more detail, $3 \mathrm{mg}$ of SG and $1 \mathrm{mg}$ of $\left(\mathrm{NH}_{4}\right)_{2} \mathrm{MoS}_{4}$ were dissolved in $3 \mathrm{~mL}$ DMF to prepare the $\mathrm{SG} / \mathrm{MoS}_{2}$ (denoted as $3: 1$, according to the weight ratio of reactants employed), while $30 \mathrm{mg}$ of SG and $10 \mathrm{mg}$ of $\left(\mathrm{NH}_{4}\right)_{2} \mathrm{WS}_{4}$ were used for the preparation of $\mathrm{SG} / \mathrm{WS}_{2}(3: 1)$. The $\mathrm{SG} / \mathrm{MoS}_{2}(1: 1)$ and $\mathrm{SG} / \mathrm{WS}_{2}(1: 1)$ hybrids were prepared accordingly. All samples were bath sonicated for 30 minutes prior to the microwave treatment. The materials were then centrifuged at $4000 \mathrm{rpm}$ for 5 minutes and washed with dimethylformamide, water and methanol several times, before characterizing and testing them as electrocatalysts.

\section{Results and discussion}

The preparation of SG was accomplished by treatment of graphene oxide with Lawesson's reagent, as illustrated in Fig. 1. Under specific conditions Lawesson's reagent acts as both

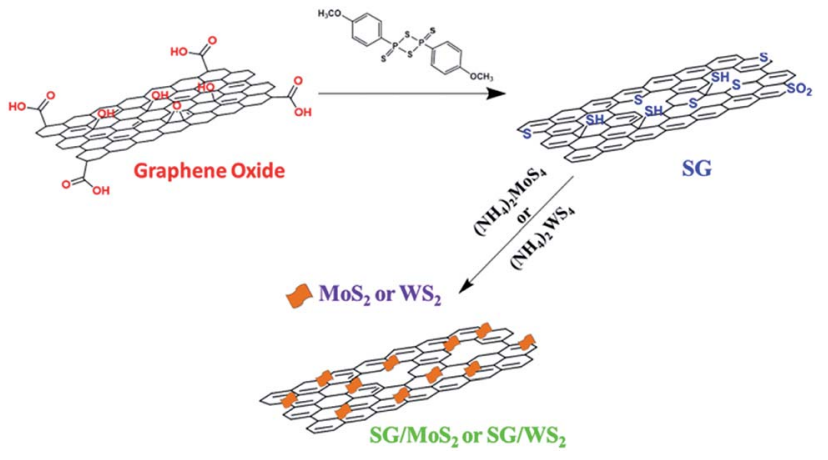

Fig. 1 Illustration of the preparation of sulfur-doped graphene (SG) with Lawesson's reagent and fabrication of SG/MoS 2 and SG/WS hererostructures via microwave irradiation.

a sulfur source and a reducing agent, ${ }^{38}$ restoring the defected graphene lattice by forming new and/or more graphene domains with continuous $\mathrm{sp}^{2}$ hybridization. Next, the SG/MoS and $\mathrm{SG} / \mathrm{WS}_{2}$ heterostructured hybrids were realized upon microwave irradiation by employing two different weight ratios of SG and ammonium tetrathiomolybdate and tetrathiotungstate, namely $3: 1$ and $1: 1$, as the inorganic precursor species for $\mathrm{MoS}_{2}$ and $\mathrm{WS}_{2}$, respectively. In particular, microwave irradiation as a green and facile method enables the preparation of nanostructures with controllable size and morphology and more active sites which make them more catalytically effective, in contrast with expensive, time-consuming and complex methods such as CVD that are commonly employed for preparing $\mathrm{MoS}_{2}$-based electrocatalysts.

Direct evidence for the realization of SG was obtained by vibrational spectroscopy and thermogravimetric analysis. In more detail, the ATR-IR spectrum of SG shows a broad band at $1080 \mathrm{~cm}^{-1}$ corresponding to $\mathrm{C}-\mathrm{S}$ vibrations (Fig. 2a), while bands related to oxygen species owing to the starting material graphene oxide and attributed to carbonyl stretching vibrations of carboxylic acid moieties at $1720 \mathrm{~cm}^{-1}$ and to ether groups at 1220 and $1050 \mathrm{~cm}^{-1}$ disappeared or were significantly reduced. Furthermore, the Raman spectrum of SG was changed as compared to that of graphene oxide. Evidently, the $\mathrm{sp}^{2}$ related G-band was shifted to lower frequencies by $8 \mathrm{~cm}^{-1}$, at $1593 \mathrm{~cm}^{-1}$ (Fig. 2b), as compared to that attributed to graphene oxide, indicating that incorporation of sulfur within the lattice of graphene results in n-doping for SG. ${ }^{39}$ Moreover, the $I_{\mathrm{D}} / I_{\mathrm{G}}$ ratio was found to increase for $S G$ as compared to that for graphene oxide. This is due to the increase of the defectiveness attributed to etching of graphene sheets, with the simultaneous formation of new and/or more graphene domains with continuous $\mathrm{sp}^{2}$ hybridization, resulting from a reduction process that also takes place along with the sulfur-doping upon treatment with Lawesson's reagent. ${ }^{38}$ The latter was further confirmed by thermogravimetric analysis (TGA), which revealed a 50\% reduction on the mass loss observed from the thermal decomposition of SG as compared to the value registered for graphene oxide. In Fig. 2c the TGA graphs of SG and graphene oxide are presented and compared. Evidently, a $10 \%$ mass loss was observed in the temperature range of $230-420{ }^{\circ} \mathrm{C}$ under an inert 

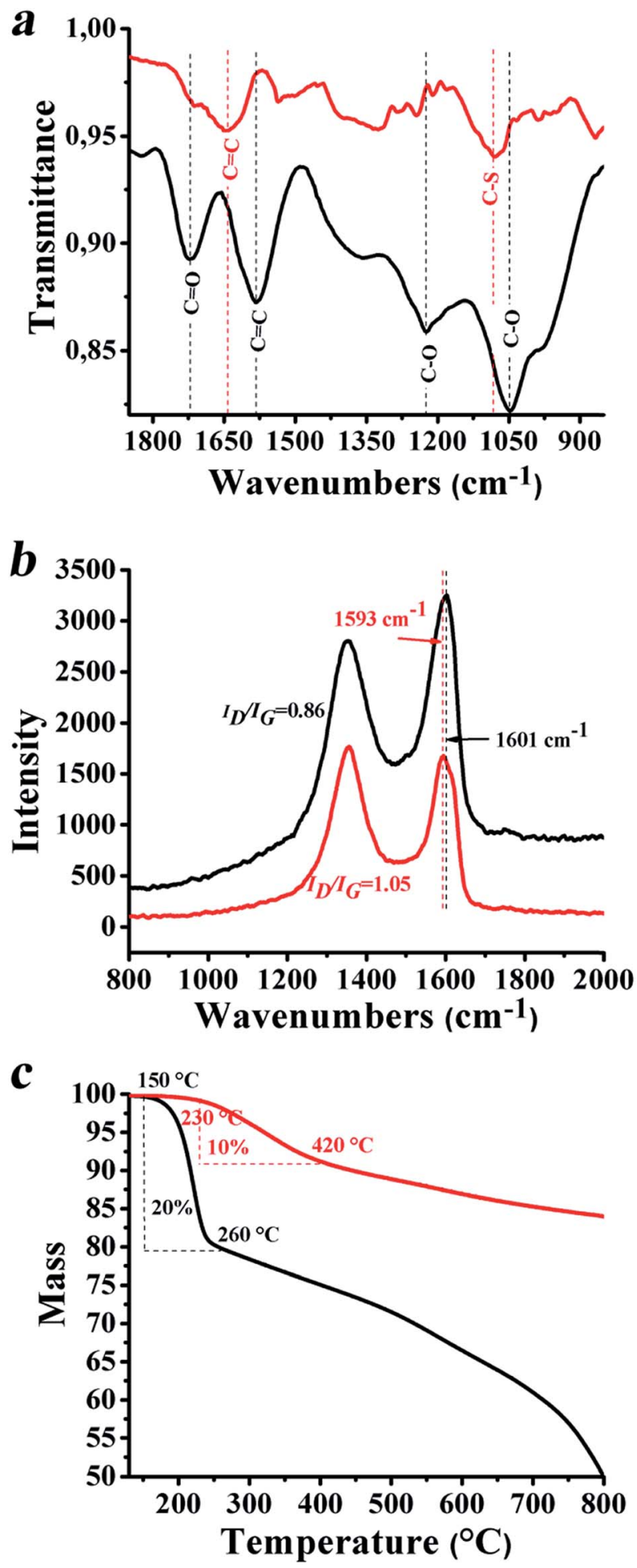

Fig. 2 (a) ATR-IR spectra of SG (red) and graphene oxide (black). (b) Raman spectra (514 nm) of SG (red) and graphene oxide (black). (c) Thermogravimetric analysis graphs of SG (red) and graphene oxide (black), obtained under nitrogen.

atmosphere for SG, as opposed to the $20 \%$ for graphene oxide in the temperature range $150-260{ }^{\circ} \mathrm{C}$. The shift to a higher decomposition temperature for SG as opposed to that for graphene oxide is justified by considering that Lawesson's reagent not only incorporated sulfur species within the graphene lattice but also reduced the oxygenated moieties, restoring to some extent the graphene network by forming islands with a continuous $\mathrm{sp}^{2}$ structure. The mass loss occurrig above $420{ }^{\circ} \mathrm{C}$ is attributed to the thermal decomposition of $\mathrm{sp}^{3}$ defects created at sites nearby where sulfur doping took place.

Focusing on $\mathrm{SG} / \mathrm{MoS}_{2}$ and $\mathrm{SG} / \mathrm{WS}_{2}$, Raman spectroscopy revealed characteristic modes due to both components, regardless of the weight ratio of $3: 1$ or $1: 1$ between the SG and the inorganic precursor species for $\mathrm{MoS}_{2}$ and $\mathrm{WS}_{2}$ employed for the preparation of the hybrids. This is to say that, for SG/MoS , bands at 379 and $405 \mathrm{~cm}^{-1}$ (Fig. 3a), corresponding to the inplane $\mathrm{E}_{2 \mathrm{~g}}^{1}$ and out-of-plane $\mathrm{A}_{1 \mathrm{~g}}$ vibrational modes of $\mathrm{MoS}_{2}$, respectively, ${ }^{\mathbf{1 , 4 0 , 4 1}}$ and for $\mathrm{SG} / \mathrm{WS}_{2}$ at 352 and $415 \mathrm{~cm}^{-1}$ (Fig. $3 \mathrm{~b}$ ), corresponding to the $\mathrm{E}_{2 \mathrm{~g}}^{1}$ and $\mathrm{A}_{1 \mathrm{~g}}$ modes of $\mathrm{WS}_{2}$, respectively, ${ }^{42}$ were observed, while in both spectra the $\mathrm{D}$ and $\mathrm{G}$ bands due to SG were present at 1352 and $1593 \mathrm{~cm}^{-1}$, respectively. The $I_{\mathrm{D}} / I_{\mathrm{G}}$ ratio for $\mathrm{SG} / \mathrm{MoS}_{2}$ and $\mathrm{SG} / \mathrm{WS}_{2}$ was found almost unaltered, $c a$. 1.08 and 1.12 respectively, as compared to that registered for SG, demonstrating that the microwave irradiation conditions employed for the growth and incorporation of $\mathrm{MoS}_{2}$ and $\mathrm{WS}_{2}$ did not cause any serious damage to the framework of SG.

Thermogravimetric analysis (TGA) assays, performed under a nitrogen atmosphere, allowed the estimation of the amount of $\mathrm{MoS}_{2}$ and $\mathrm{WS}_{2}$ within the hybrid materials. Specifically, SG/
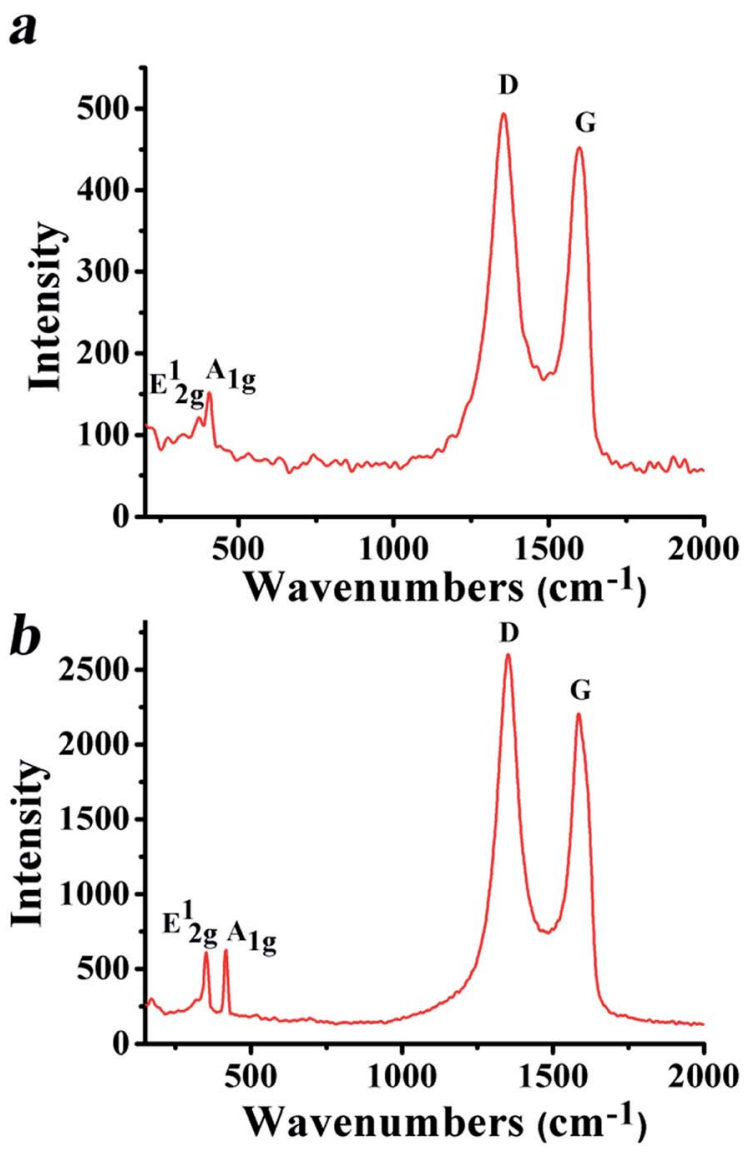

Fig. 3 Raman spectra $(514 \mathrm{~nm}$ ) of (a) SG/MoS 2 (3:1) and (b) SG/WS $(3: 1)$ heterostructured hybrid materials. 
$\mathrm{MoS}_{2}(3: 1)$ and SG/MoS $(1: 1)$ were found to thermally lose 22 and $26 \%$ of mass, respectively, in the temperature range of $200-$ $800{ }^{\circ} \mathrm{C}$ (ESI, Fig. S1a $\dagger$ ). Based on the latter observation and considering that SG is also thermally labile, showing around $10 \%$ mass loss in the temperature range of $230-420{ }^{\circ} \mathrm{C}$ and another $6 \%$ up to $800{ }^{\circ} \mathrm{C}$ (Fig. 2), while intact $\mathrm{MoS}_{2}$ shows a continuous mass loss over the whole temperature range (ESI, Fig. S1a $\dagger$ ) due to the presence of defect sites, the rough content of $\mathrm{MoS}_{2}$ within SG/MoS (3:1) and SG/MoS $(1: 1)$ was estimated to be 37 and $66 \%$, respectively. Similarly, from the TGA profiles for SG/WS $(3: 1)$ and SG/WS $(1: 1)$ (ESI, Fig. S1b $\dagger$ ), while taking into account the overall $16 \%$ mass loss due to SG, the content of $\mathrm{WS}_{2}$ in the two hybrids was estimated to be 35 and $64 \%$, respectively.

Fig. 4 shows HR-TEM images and EEL spectra of intact $\mathrm{MoS}_{2}$ and $\mathrm{WS}_{2}$ as well as of SG/MoS ${ }_{2}$ and SG/WS $/ \mathrm{W}_{2}$ hybrids for the two different weight ratios $3: 1$ and $1: 1$ screened. Intact $\mathrm{MoS}_{2}$ and $\mathrm{WS}_{2}$ showed different nanostructures with different crystallographic orientations, aggregated in clusters with size in tenths of nm. This behavior can be clearly seen in the FFT diffraction pattern of $\mathrm{MoS}_{2}$ (inset of Fig. 4a), where several defined spots can be observed. As for the SG/MoS 2 and $\mathrm{SG} / \mathrm{WS}_{2}$, the TEM analysis showed a variation in contrast, which is believed to be related to the presence of $\mathrm{MoS}_{2}$ and $\mathrm{WS}_{2}$, respectively, on SG see for example the crumpled structures in Fig. 4d. The EELS results, shown in Fig. S2a, S2b1 and S2b2 $\uparrow$ validated the presence of $\mathrm{MoS}_{2}$ and $\mathrm{WS}_{2}$ in SG, which is coherent with the rest of the microscopy and spectroscopic studies performed. In addition, SEM imaging of SG (ESI, Fig. S3†) together with SEM and EDX analysis for $\mathrm{MoS}_{2}$, SG/MoS 2 and SG/WS (ESI, Fig. S4 and S5 $\dagger$ ) and TEM imaging (ESI, Fig. S6 $\dagger$ ) further confirmed the presence of $\mathrm{MoS}_{2}$ and $\mathrm{WS}_{2}$ in all the hybrid materials.

Next, the electrocatalytic activity of SG/MoS 2 and SG/WS toward the HER was examined by performing linear sweep

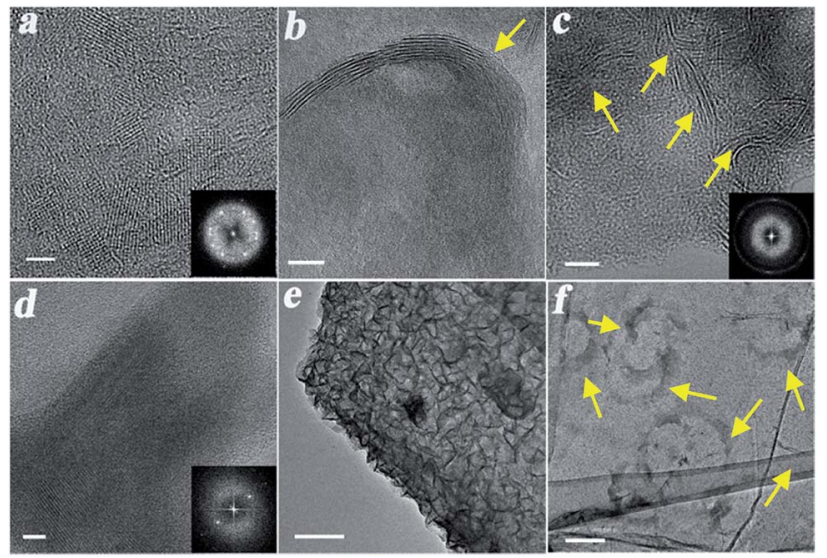

Fig. 4 HR-TEM images of (a) $M_{2} S_{2}$ deposited on an amorphous carbon substrate, (b) SG/MoS 2 (1:1), and (c) SG/MoS 2 (3:1). HR-TEM images of (d) $W S_{2}$ on an amorphous carbon substrate, (e) SG/WS (1: 1), and (f) SG/WS 2 (3: 1). Scale bars: (a) $2 \mathrm{~nm}$, (b) $10 \mathrm{~nm}$, (c) $5 \mathrm{~nm}$, (d) $5 \mathrm{~nm}$, (e) $100 \mathrm{~nm}$ and (f) $100 \mathrm{~nm}$. Some of the most representative $\mathrm{MoS}_{2}$ and $\mathrm{WS}_{2}$ nanostructures are highlighted with yellow arrows in (b), (c) and (f), respectively. voltammetry measurements with a rotating-disc working glassy carbon electrode in a standard three-electrode glass cell at a scan rate of $5 \mathrm{mV} \mathrm{s}^{-1}$ in nitrogen saturated $0.5 \mathrm{M}$ aqueous sulfuric acid. Polarization curves for the SG/MoS 2 (3:1) and SG/ $\mathrm{MoS}_{2}$ (1:1) hybrids, accordingly denoted for the different weight ratios of SG and the inorganic precursor species for $\mathrm{MoS}_{2}$ employed for the preparation, together with those for individual $\mathrm{MoS}_{2}$ and SG along with that for commercially available Pt/C as a reference are shown in Fig. 5a. Evidently, for a given potential, the cathodic current increased for SG/MoS as compared to that for individual $\mathrm{MoS}_{2}$ and SG. The onset overpotential for SG/ $\mathrm{MoS}_{2}(1: 1)$ was registered at $-0.175 \mathrm{~V} v s$. RHE, and shifted by $0.215 \mathrm{~V}$ to a more positive potential as compared to that for individual $\mathrm{MoS}_{2}$ appearing at $-0.390 \mathrm{~V} v s$. RHE. Notably, for the electrocatalyst with a higher amount of SG, namely SG/MoS $(3: 1)$, the onset overpotential is similar to that of commercially available Pt/C, appearing at $-0.026 \mathrm{~V}$ vs. RHE (Fig. 5a). The same trend was identified for $\mathrm{SG} / \mathrm{WS}_{2}$, in which the heterostructured hybrid with the higher amount of SG, namely SG/ $\mathrm{WS}_{2}$ (3:1), showed lower overpotential, ca. $-0.140 \mathrm{~V} v s$. RHE as compared not only with that of the individual $\mathrm{WS}_{2}, c a .-0.460 \mathrm{~V}$, but also with that of SG/WS 2 (1: 1), ca. $-0.390 \mathrm{~V}$ (Fig. 5b). An overall graph showing the onset overpotential for all examined materials is presented in Fig. 5c. The aforementioned results not only highlight the beneficial role of SG as the substrate for uniformly immobilizing $\mathrm{MoS}_{2}$ and $\mathrm{WS}_{2}$, but also more importantly stress the importance of the amount of SG relative to $\mathrm{MoS}_{2}$ and $\mathrm{WS}_{2}$ for achieving optimum electrocatalytic performance toward the HER. Overall, a synergetic effect attributed to both the conductive nature of SG in intimate contact with $\mathrm{MoS}_{2}$ and $\mathrm{WS}_{2}$, allowing effective charge transport, and the presence
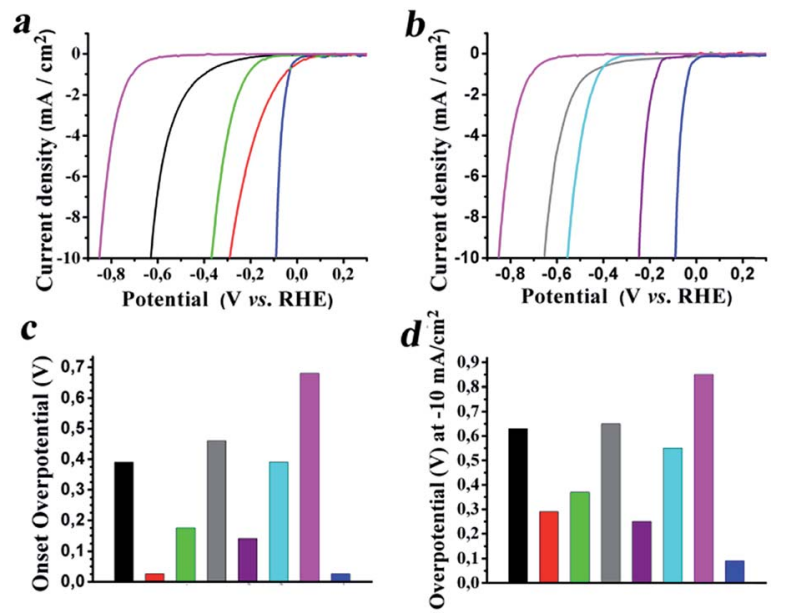

Fig. 5 Linear sweep voltammograms (LSV) for the HER of (a) SG/MoS (3:1) (red), SG/MoS 2 (1:1) (green), MoS (black), SG (pink) and Pt/C (blue), and (b) SG/WS 2 (3:1) (purple), SG/WS 2 (1:1) (cyan), WS 2 (grey), $\mathrm{SG}$ (pink) and Pt/C (blue). The LSV polarization curves were obtained in a nitrogen saturated aqueous $0.5 \mathrm{M} \mathrm{H}_{2} \mathrm{SO}_{4}$ electrolyte, at a rotation speed of $1600 \mathrm{rpm}$ and scan rate of $5 \mathrm{mV} \mathrm{s}^{-1}$. (c) Graph for onset overpotential for all the tested materials. (d) Graph for overpotential registered at a $-10 \mathrm{~mA} \mathrm{~cm}{ }^{-2}$ current density for all the tested materials. 
of active edge sites in $\mathrm{MoS}_{2}$ and $\mathrm{WS}_{2}$, leads to more active electrocatalytic behavior towards the HER with comparable performance with that of platinum. Focusing on the best performing hybrids as electrocatalysts for the HER, namely SG/ $\mathrm{MoS}_{2}(3: 1)$ and SG/WS $(3: 1)$, bubbles of hydrogen were observed to evolve at a cathodic current density of 0.6 and $0.3 \mathrm{~mA} \mathrm{~cm} \mathrm{~cm}^{-2}$, respectively, with enhanced rates at around -0.290 and $-0.240 \mathrm{~V}$, respectively. Considering that the amount of cathodic current density is proportional to the amount of hydrogen evolved and since it is very common to compare electrocatalysts for the HER against overpotentials at a cathodic current density of $10 \mathrm{~mA} \mathrm{~cm} \mathrm{~cm}^{-2}$, the corresponding tabulated graph for all the examined materials, shown in Fig. 5d, nicely demonstrates the highest activity for SG/MoS $(3: 1)$ and $\mathrm{SG} / \mathrm{WS}_{2}(3: 1)$, with the former performing best at lower overpotentials ( $c f$. Fig. $5 \mathrm{c})$.

In order to obtain meaningful insights and characterize the charge transport efficiency and efficacy of the electrocatalytic HER, Tafel slopes for SG/MoS 2 (3:1), SG/MoS 2 (1:1), SG/WS $(3: 1)$ and $\mathrm{SG} / \mathrm{WS}_{2}(1: 1)$ were extracted from the LSV polarization curves and presented in Fig. $6 \mathrm{a}$ and $\mathrm{b}$, respectively. In addition, the tabulated Tafel values are given in Fig. 6c. Generally, the Tafel slope is an inherent property of the electrocatalyst that is determined from the rate-limiting step of the HER. Hence, analysis of the Tafel plot data aids in the elucidation of the HER mechanism and more importantly in the identification of the rate-limiting step of the reaction. With all this in mind, the dominant mechanism of hydrogen evolution for the materials tested is interpreted by considering initial adsorption of a proton onto the electrode surface via a reduction process, according to Volmer adsorption - eqn (1). Next, bonding of the adsorbed hydrogen with a proton and electron transfer from the electrode surface takes place, according to Heyrovsky desorption - eqn (2). Alternatively, recombination of two hydrogen atoms adsorbed on the electrode surface occurs, according to Tafel desorption - eqn (3).

$$
\begin{gathered}
\mathrm{H}_{3} \mathrm{O}^{+}+\mathrm{e}^{-} \rightarrow \mathrm{H}_{\mathrm{ads}}+\mathrm{H}_{2} \mathrm{O} \\
\mathrm{H}_{\mathrm{ads}}+\mathrm{H}_{3} \mathrm{O}^{+}+\mathrm{e}^{-} \rightarrow \mathrm{H}_{2}+\mathrm{H}_{2} \mathrm{O} \\
\mathrm{H}_{\mathrm{ads}}+\mathrm{H}_{\mathrm{ads}} \rightarrow \mathrm{H}_{2}
\end{gathered}
$$

Individual $\mathrm{MoS}_{2}, \mathrm{WS}_{2}$ and SG exhibited relatively high Tafel slopes, 271, 246 and $153 \mathrm{mV} \mathrm{dec}{ }^{-1}$, respectively. Realization of the $\mathrm{SG} / \mathrm{MoS}_{2}$ and $\mathrm{SG} / \mathrm{WS}_{2}$ heterostructured hybrids, regardless of the relative amount of $\mathrm{SG}$ versus the transition metal precursor employed, i.e. $3: 1$ and $1: 1$, caused a drop in the corresponding Tafel value (Fig. 6). Since a smaller Tafel slope implies that for the generation of an equivalent current only a lower overpotential is required, the electrocatalytic activity of $\mathrm{MoS}_{2}$ and $\mathrm{WS}_{2}$ was improved, particularly for the SG/MoS (3:1) and $\mathrm{SG} / \mathrm{WS}_{2}(3: 1)$ systems, which showed Tafel slopes of 152 and $53 \mathrm{mV} \mathrm{dec}^{-1}$, respectively. Again, the improvement in charge transport is facilitated by the good electrical contact between SG and TMDs, while the observed Tafel values manifest
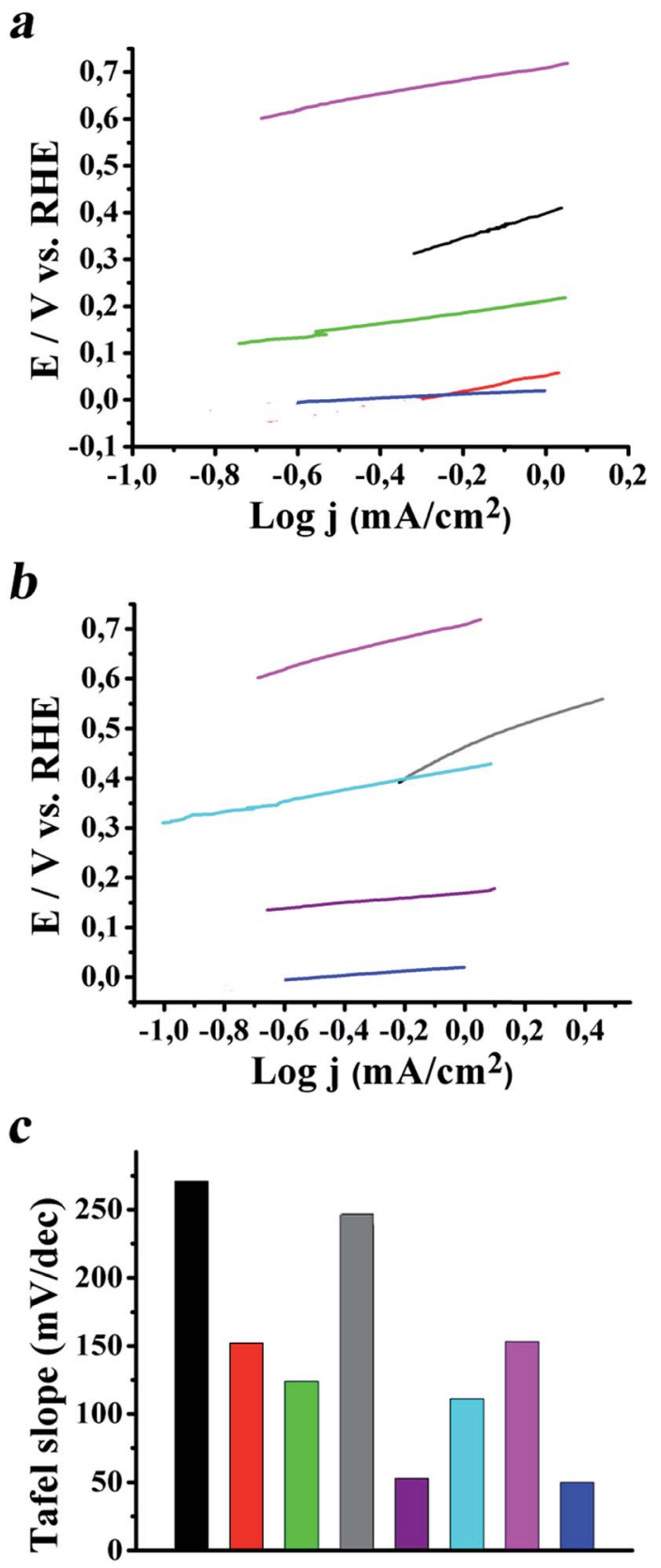

Fig. 6 Tafel slopes for (a) SG/MoS $(3: 1)$ (red), SG/MoS 2 (1:1) (green), $\mathrm{MoS}_{2}$ (black), SG (pink) and Pt/C (blue), and (b) SG/WS 2 (3:1) (purple), $S G / W S_{2}(1: 1)$ (cyan), WS 2 (grey), SG (pink) and Pt/C (blue), showing overpotential versus current density. (c) Tabulated values for Tafel slopes for all the tested materials.

that the adsorption of hydrogen onto the modified electrode is the rate-limiting step for the $\mathrm{SG} / \mathrm{MoS}_{2}(3: 1)$ according to eqn (1), while for the $\mathrm{SG} / \mathrm{WS}_{2}(3: 1)$ the electrochemical desorption of adsorbed hydrogen atoms onto the modified electrode to generate hydrogen is the rate-limiting step, according to eqn (2).

In order to further understand the improved electrocatalytic activity of the SG/MoS 2 and SG/WS $\mathrm{WS}_{2}$ hybrid materials, the electrochemically active surface area (ECSA) was calculated according to the equation ECSA $=C_{\mathrm{dl}} / C_{\mathrm{s}}$, where $C_{\mathrm{dl}}$ is the electrochemical double-layer capacitance and $C_{\mathrm{s}}$ is the specific 
Table 1 Electrochemical parameters for the HER

\begin{tabular}{|c|c|c|c|c|}
\hline Catalyst & $\begin{array}{l}\text { Onset } \\
\text { overpotential } \\
\text { (V vs. } \mathrm{RHE})\end{array}$ & $\begin{array}{l}\text { Overpotential } \\
(V v s . \mathrm{RHE}) \text { at } \\
-10 \mathrm{~mA} \mathrm{~cm}^{-2}\end{array}$ & $\begin{array}{l}\text { Tafel slope } \\
\left(\mathrm{mV} \mathrm{dec}^{-1}\right)\end{array}$ & $\begin{array}{l}\text { ECSA } \\
\left(\mathrm{cm}^{2}\right)\end{array}$ \\
\hline $\mathrm{MoS}_{2}$ & 0.39 & 0.63 & 271 & $\sim 2.25$ \\
\hline $\operatorname{MoS}_{2}{ }^{a}$ & 0.39 & 0.69 & 240 & - \\
\hline $\mathrm{SG} / \mathrm{MoS}_{2}(3: 1)$ & 0.026 & 0.29 & 152 & $\sim 15.4$ \\
\hline $\begin{array}{l}\mathrm{SG} / \mathrm{MoS}_{2} \\
(3: 1)^{a}\end{array}$ & 0.033 & 0.30 & 160 & - \\
\hline $\mathrm{SG} / \mathrm{MoS}_{2}(1: 1)$ & 0.175 & 0.37 & 124 & $\sim 6.9$ \\
\hline $\begin{array}{l}\mathrm{SG} / \mathrm{MoS}_{2} \\
(1: 1)^{a}\end{array}$ & 0.175 & 0.41 & 111 & - \\
\hline $\mathrm{WS}_{2}$ & 0.46 & 0.65 & 246 & $\sim 9.75$ \\
\hline $\mathrm{WS}_{2}{ }^{a}$ & 0.47 & 0.68 & 213 & - \\
\hline $\mathrm{SG} / \mathrm{WS}_{2}(3: 1)$ & 0.14 & 0.25 & 53 & $\sim 20$ \\
\hline $\mathrm{SG} / \mathrm{WS}_{2}(3: 1)^{a}$ & 0.15 & 0.26 & 56 & - \\
\hline SG/WS 2 (1: 1) & 0.39 & 0.55 & 111 & $\sim 13.87$ \\
\hline $\mathrm{SG} / \mathrm{WS}_{2}(1: 1)^{a}$ & 0.39 & 0.57 & 113 & - \\
\hline SG & 0.68 & 0.85 & 153 & $\sim 7.25$ \\
\hline $\mathrm{SG}^{a}$ & 0.68 & 0.87 & 157 & - \\
\hline $\mathrm{Pt} / \mathrm{C}$ & 0.026 & 0.09 & 50 & - \\
\hline $\mathrm{Pt} / \mathrm{C}^{a}$ & 0.054 & 0.096 & 50 & - \\
\hline
\end{tabular}

capacitance of a flat surface with $1 \mathrm{~cm}^{2}$ real surface area with a value assumed to be $40 \mu \mathrm{F} \mathrm{cm}^{-2}$ for the flat electrode. ${ }^{43}$ To this end, measuring cyclic voltammograms in a non-faradaic region at different scan rates of 50,100, 200,300, 400 and $500 \mathrm{mV} \mathrm{s}^{-1}$ (ESI, Fig. S7 $\dagger$ ), allowed the estimation of the ECSA value from the $C_{\mathrm{dl}}$ by plotting the $\Delta j=\left(j_{\mathrm{a}}-j_{\mathrm{c}}\right)$ at $0.1 \mathrm{~V} v s$. RHE as a function of the scan rate according to the equation $C_{\mathrm{dl}}=\mathrm{d}(\Delta j) / 2 \mathrm{~d} V_{\mathrm{b}} \cdot{ }^{43}$ Evidently, the ECSA values of SG/MoS $(3: 1)$ and SG/WS $2(3: 1)$ were significantly higher than those of SG/MoS $(1: 1)$ and SG/WS 2 (1:1), respectively, as shown in Table 1 . These higher ECSA values indicate more effective accessibility of the active sites of the SG/MoS $(3: 1)$ and SG/WS $2(3: 1)$ hybrid materials, similar to recent reports. ${ }^{44}$

Finally, the long-term stability of the hybrid materials was evaluated. Durability studies as an important way to assess the electrocatalytic activity of the materials were performed (ESI, Fig. S8 $\dagger$ ) and it was found that all the tested materials exhibited high stability after cycling continuously for 1000 cycles, with negligible loss of cathodic current. In Table 1, the various HER parameters of all the screened materials, before and after 1000 cycles, are summarized.

\section{Conclusions}

In summary, the fabrication of $\mathrm{SG} / \mathrm{MoS}_{2}$ and $\mathrm{SG} / \mathrm{WS}_{2}$ hybrids, with two different weight ratios between the SG and the inorganic species as $3: 1$ and $1: 1$, as efficient electrocatalysts for the HER was accomplished. The preparation of the hybrids involved microwave-assisted thermal decomposition of $\left(\mathrm{NH}_{4}\right)_{2} \mathrm{MoS}_{4}$ and $\left(\mathrm{NH}_{4}\right)_{2} \mathrm{WS}_{4}$, employed as inorganic precursors for $\mathrm{MoS}_{2}$ and $\mathrm{WS}_{2}$, respectively, in the presence of SG. Complementary spectroscopic characterization based on Raman spectroscopy and
HR-TEM coupled with EELS confirmed the successful formation of SG/MoS 2 and SG/WS . These heterostructured hybrids were scrutinized for the HER and it was found that the presence of SG not only significantly improved the catalytic activity of $\mathrm{MoS}_{2}$ and $\mathrm{WS}_{2}$ but also made it comparable to that of the commercial Pt/C catalyst. This is in line with recent results based on N-doped graphene employed as a substrate for TMDs, ${ }^{33,34}$ highlighting the beneficial role of heteroatom doping in graphene for improving the electrocatalytic activity towards the HER. More precisely, based on LSV assays, the best electrocatalytic activity for the HER was found for hybrids with a higher content of SG, namely SG/MoS $2(3: 1)$ and SG/WS $2(3: 1)$. The uniform and robust immobilization of $\mathrm{MoS}_{2}$ and $\mathrm{WS}_{2}$ on the surface of SG, without the involvement of surfactants and any insulating organic moieties, thereby facilitating an effective chargetransport processes due to intimate contact, plays a crucial role in the exceptional electrocatalytic functioning of the SG/ $\mathrm{MoS}_{2}$ and SG/WS${ }_{2}$ hybrids. In addition, the presence of active edge sites in $\mathrm{MoS}_{2}$ and $\mathrm{WS}_{2}$, in combination with the novel characteristics of SG, possesses its own share on the better electrocatalytic behavior of SG/MoS 2 and $\mathrm{SG} / \mathrm{WS}_{2}$ against the HER. The higher ECSA values for the hybrid heterostructured materials with the higher content of SG, namely SG/MoS $2(3: 1)$ and SG/WS $(3: 1)$, provided a large functioning surface area of catalytic active sites, excellent hydrogen production ability, and hence superior catalytic performance. Furthermore, based on the Tafel plot derived from the corresponding polarization curves, the adsorption of hydrogen as the rate-limiting step for $\mathrm{SG} / \mathrm{MoS}_{2}(3: 1)$ was revealed, while for SG/WS $2(3: 1)$ the kinetics was governed by the electrochemical desorption of adsorbed hydrogen atoms to generate hydrogen. Overall, considering that this particular facile approach for developing SG/MoS ${ }_{2}$ and SG/ $\mathrm{WS}_{2}$ heterostructured hybrids can be extended to fabricate diverse materials, incorporating other transition metals and different chalcogens, we firmly believe that a plethora of viable electrocatalysts can be produced, opening wide avenues in the areas of energy conversion and storage.

\section{Conflicts of interest}

There are no conflicts to declare.

\section{Acknowledgements}

This project has received funding from the European Union's Horizon 2020 research and innovation programme under the Marie Sklodowska-Curie grant agreement No. 642742. Partial support of this work by the project "Advanced Materials and Devices" (MIS 5002409) which is implemented under the "Action for the Strategic Development on the Research and Technological Sector", funded by the Operational Program "Competitiveness, Entrepreneurship and Innovation" (NSRF 2014-2020) and co-financed by Greece and the European Union (European Regional Development Fund) is also acknowledged. The HR-TEM and EELS studies were conducted at the Laboratorio de Microscopias Avanzadas, Instituto de Nanociencia de Aragon, Universidad de Zaragoza, Spain. R. A. gratefully 
acknowledges the support from the Spanish Ministry of Economy and Competitiveness (MINECO) through project MAT2016-79776-P (AEF/FEDER. UE), the Government of Aragon and ESF under the project "Construyendo Europa desde Aragon” 2014-2020 (grant number E13_17R), and from the EU H2020 program "Graphene Flagship" (Grant Agreement 785219).

\section{References}

1 G. Pagona, C. Bittencourt, R. Arenal and N. Tagmatarchis, Chem. Commun., 2015, 51, 12950-12953.

2 J. Zheng, H. Zhang, S. Dong, Y. Liu, C. T. Nai, H. S. Shin, H. Y. Jeong, B. Liu and K. P. Loh, Nat. Commun., 2014, 5, 2995.

3 Y. Yao, L. Torentino, Z. Yang, X. Song, W. Zhang, Y. Chen and C. Wong, Adv. Funct. Mater., 2013, 23, 3577-3583.

4 G. Eda, H. Yamaguchi, D. Voiry, T. Fujita, M. Chen and M. Chhowalla, Nano Lett., 2011, 11, 5111-5116.

5 J. N. Coleman, M. Lotya, A. O'Neill, S. D. Bergin, P. J. King, U. Khan, K. Young, A. Gaucher, S. De, R. J. Smith, I. V. Shvets, S. K. Arora, G. Stanton, H. -Y. Kim, K. Lee, G. T. Kim, G. S. Duesberg, T. Hallam, J. J. Boland, J. J. Wang, J. F. Donegan, J. C. Grunlan, G. Moriarty, A. Shmeliov, R. J. Nicholls, J. M. Perkins, E. M. Grieveson, K. Theuwissen, D. W. McComb, P. D. Nellist and V. Nicolosi, Science, 2011, 331, 568-571.

6 R. Ganatra and Q. Zhang, ACS Nano, 2014, 8, 4074-4099.

7 R. Lv, J. A. Robinson, R. E. Schaak, D. Sun, Y. Sun, T. E. Mallouk and M. Terrones, Acc. Chem. Res., 2015, 48, 56-64.

8 Q. H. Wang, K. Kalantar-Zadeh, A. Kis, J. N. Coleman and M. S. Strano, Nat. Nanotechnol., 2012, 7, 699-712.

9 G. Zhang, H. Liu, J. Qu and J. Li, Energy Environ. Sci., 2016, 9, 1190-1209.

10 D. Voiry, J. Yang and M. Chhowalla, Adv. Mater., 2016, 28, 6197-6206.

11 H. Li, C. Tsai, A. L. Koh, L. Cai, A. W. Contryman, A. H. Fragapane, J. Zhao, H. S. Han, H. C. Manoharan, F. Abild-Pedersen, J. K. Norskov and X. Zheng, Nat. Mater., 2015, 15, 48-53.

12 X. Chia, A. Y. S. Eng, A. Ambrosi, S. M. Tan and M. Pumera, Chem. Rev., 2015, 115, 11941-11966.

13 L. Cheng, W. Huang, Q. Gong, C. Liu, Z. Liu, Y. Li and H. Dai, Angew. Chem., Int. Ed., 2014, 53, 7860-7863.

14 D. Voiry, H. Yamaguchi, J. Li, R. Silva, D. C. B. Alves, T. Fujita, M. Chen, T. Asefa, V. B. Shenoy, G. Eda and M. Chhowalla, Nat. Mater., 2013, 12, 850-855.

15 D. Voiry, M. Salehi, R. Silva, T. Fujita, M. Chen, T. Asefa, V. B. Shenoy, G. Eda and M. Chhowalla, Nano Lett., 2013, 13, 6222-6227.

16 M. A. Lukowski, A. S. Daniel, F. Meng, A. Forticaux, L. Li and S. Jin, J. Am. Chem. Soc., 2013, 135, 10274-10277.

17 J. Kibsgaard, Z. Chen, B. N. Reinecke and T. F. Jaramillo, Nat. Mater., 2012, 11, 963-969.

18 Y. Li, H. Wang, L. Xie, Y. Liang, G. Hong and H. Dai, J. Am. Chem. Soc., 2011, 133, 7296-7299.
19 T. F. Jaramillo, K. P. Jorgensen, J. Bonde, J. H. Nielsen, S. Horch and I. Chorkendorff, Science, 2007, 317, 100-102.

20 Y. Yang, H. Fei, G. Ruan, C. Xiang and J. M. Tour, Adv. Mater., 2014, 26, 8163-8168.

21 B. Guo, K. Yu, H. Li, H. Song, Y. Zhang, X. Lei, H. Fu, Y. Tan and Z. Zhu, ACS Appl. Mater. Interfaces, 2016, 8, 5517-5525.

22 H. Li, H. Wu, S. Yuan and H. Qian, Sci. Rep., 2016, 6, 21171.

23 K. D. Rasamani, F. Alimohammadi and Y. Sun, Mater. Today, 2017, 20, 83-91.

24 D. K. Perivoliotis and N. Tagmatarchis, Carbon, 2017, 118, 493-510.

25 L. Liao, J. Zhu, X. Bian, L. Zhu, M. D. Scanlon, H. H. Girault and B. Liu, Adv. Funct. Mater., 2013, 23, 5326-5333.

26 L. Yang, W. Zhou, J. Lu, D. Hou, Y. Ke, G. Li, Z. Tang, X. Kang and S. Chen, Nano Energy, 2016, 22, 490-498.

27 Y. Sun, F. Alimohammadi, D. Zhang and G. Guo, Nano Lett., 2017, 17, 1963-1969.

28 M. Chatti, T. Gengenbach, R. King, L. Spiccia and A. N. Simonov, Chem. Mater., 2017, 29, 3092-3099.

29 A. Kagkoura, T. Skaltsas and N. Tagmatarchis, Chem.-Eur. J., 2017, 23, 12967-12979.

30 Y. Jieun, V. Damien, A. S. Joon, K. Dongwoo, K. A. Young, C. Manish and S. H. Suk, Angew. Chem., Int. Ed., 2013, 52, 13751-13754.

31 X. Zheng, J. Xu, K. Yan, H. Wang, Z. Wang and S. Yang, Chem. Mater., 2014, 26, 2344-2353.

32 E. G. S. Firmiano, M. A. L. Cordeiro, A. C. Rabelo, C. J. Dalmaschio, A. N. Pinheiro, E. C. Pereira and E. R. Leite, Chem. Commun., 2012, 48, 7687-7689.

33 H. Dong, C. Liu, H. Ye, L. Hu, B. Fugetsu, W. Dai, Y. Cao, X. Qi, H. Lu and X. Zhang, Sci. Rep., 2015, 5, 17542.

34 Y. J. Tang, Y. Wang, X. L. Wang, S. L. Li, W. Huang, L. Z. Dong, C. H. Liu, Y. F. Li and Y. Q. Lan, Adv. Energy Mater., 2016, 6, 1600116.

35 P. H. Ling and P. Martin, ChemElectroChem, 2015, 2, 190199.

36 I.-Y. Jeon, S. Zhang, L. Zhang, H.-J. Choi, J.-M. Seo, Z. Xia, L. Dai and J.-B. Baek, Adv. Mater., 2013, 25, 6138-6145.

37 X. Wang, G. Sun, P. Routh, D.-H. Kim, W. Huang and P. Chen, Chem. Soc. Rev., 2014, 43, 7067-7098.

38 D. K. Perivoliotis, Y. Sato, K. Suenaga and N. Tagmatarchis, ACS Appl. Energy Mater., 2018, 1, 3869-3880.

39 H. Liu, Y. Liu and D. Zhu, J. Mater. Chem., 2011, 21, 33353345.

40 H. Li, Q. Zhang, C. C. R. Yap, B. K. Tay, T. H. T. Edwin, A. Olivier and D. Baillargeat, Adv. Funct. Mater., 2012, 22, 1385-1390.

41 S.-L. Li, H. Miyazaki, H. Song, H. Kuramochi, S. Nakaharai and K. Tsukagoshi, ACS Nano, 2012, 6, 7381-7388.

42 H. Terrones, E. D. Corro, S. Feng, J. M. Poumirol, D. Rhodes, D. Smirnov, N. R. Pradhan, Z. Lin, M. A. T. Nguyen, A. L. Elías, T. E. Mallouk, L. Balicas, M. A. Pimenta and M. Terrones, Sci. Rep., 2014, 4, 4215.

43 F. Shong and H. Hu, Nat. Commun., 2014, 5, 4477.

44 B. You, N. Jiang, M. Sheng, W. Bhushan and Y. Sun, ACS Catal., 2016, 6, 714-722. 